\title{
Magnetic field-induced effects on NMR properties
}

\author{
J. Jokisaari,* A. M. Kantola, and J. Vaara \\ NMR Research Unit, P.O.Box 3000, FI-90014 University of Oulu, Finland
}

\begin{abstract}
In principle, all the NMR observables, spin-spin coupling $J$, nuclear shielding $\sigma$ and quadrupole coupling $\boldsymbol{q}$, are magnetic field-dependent. The field dependence may be classified into two categories: direct and indirect (apparent) dependence. The former arises from the magnetic field-induced deformation of the molecular electronic cloud, while the latter stems from a slightly anisotropic orientation distribution of molecules, due to the interaction between the anisotropy of the molecular susceptibility tensor and the external magnetic field. Here we use 1,3,5- $\mathrm{D}_{3}$-benzene as a model system to investigate the indirect effect on the one-bond ${ }^{1} \mathrm{H}-{ }^{13} \mathrm{C}$ and ${ }^{2} \mathrm{H}-{ }^{13} \mathrm{C}$ spin-spin couplings ( $J$ couplings) and the ${ }^{2} \mathrm{H}$ quadrupole coupling. Experiments carried out at four magnetic fields (4.7, 9.4, 14.1, and 18.8 Tesla) show that the indirect effect is significant already at the magnetic fields commonly used in NMR spectrometers. A joint fit of the data extracted at the different field strengths provides experimental results for the susceptibility anisotropy, ${ }^{2} \mathrm{H}$ quadrupole coupling constant and the related asymmetry parameter as well as the one-bond $\mathrm{CH}$ and $\mathrm{CD}$ coupling constants extrapolated to vanishing field strength. The field-induced contributions are found to exceed the commonly assumed error margins of the latter. The data also indicate a primary isotope effect on the one-bond $\mathrm{CH}$ coupling constant. There is a tendency to further increase the magnetic field of NMR spectrometers, which leads to more pronounced indirect contributions and eventually significant direct effects as well.
\end{abstract}


Keywords: Isotropic liquid, molecular orientation, diamagnetic anisotropy, isotope effect, 1,3,5-D3benzene

*Corresponding author

Emails: Jukka.Jokisaari@oulu.fi, Anu.Kantola@oulu.fi, juha.vaara@iki.fi, 


\section{Introduction}

Generally the nuclear magnetic resonance (NMR) parameters, nuclear shielding, spin-spin coupling and quadrupole splitting are considered independent of the applied magnetic field. However, as early as 1970, Ramsey ${ }^{1}$ published a theoretical study in which he concluded that "in some molecules, especially where the magnetic shielding is abnormally large, it is possible that the field dependence of the shielding could be large enough to be observable". Inspired by this prediction, Bendall and Doddrell carried out ${ }^{59} \mathrm{Co}$ NMR experiments of some cobalt compounds and found a field dependence of shielding in $\mathrm{Co}\left({ }^{15} \mathrm{NH}_{3}\right) \mathrm{Cl}_{3}$ and $\mathrm{Co}(\text { acac })_{3} .{ }^{2}$ About 25 years later, Manninen and Vaara presented a theory and computed from first principles the field-dependence of $-6 \times 10^{-3} \mathrm{ppmT}^{-2}$ in $\operatorname{Co}(\text { acac })_{3}$, which is an order of magnitude smaller than the experimentally derived value. ${ }^{3}$ Boyd $e t$ $a l$. , in turn, computed field dependence of the order of $10^{-5} \mathrm{ppmT}^{-2}$ for nitrogen shielding in some nitroso and isodiazene compounds. ${ }^{4}$ Similarly to nuclear shielding, the spin-spin coupling can be expected to be field-dependent as well. The semi-empirical study by Raynes and Stevens suggested that the coupling varies as $0.5 \times 10^{-5} \mathrm{HzT}^{-2}$ for "strongly magnetic nuclei in large molecules". 5 There is, however, no experimental evidence of the field dependence of spin-spin couplings.

The only experimentally observed and computationally confirmed field dependence of an NMR parameter is that of ${ }^{131}$ Xe quadrupole coupling. ${ }^{6,7,8,9}$ The ${ }^{131}$ Xe NMR spectrum consists of a 3:4:3 triplet when the electric quadrupole moment interacts with a non-zero electric field gradient (EFG) at the nuclear site. The non-zero EFG stems from the slight deformation of the electron cloud induced by the magnetic field. Apart from the direct magnetic field effect on the NMR parameters, discussed above, there may appear also an indirect (aka apparent) contribution due to the fieldinduced partial orientation of molecules. The partial orientation leads to incomplete averaging of the anisotropic parts of the NMR tensors. In other words, dipole-dipole couplings, quadrupole couplings, and the anisotropies of spin-spin coupling and chemical shift affect the observables. Such effects were 
detected already in the mid-1980's for the ${ }^{2} \mathrm{H}$ quadrupole coupling in substituted benzenes, pyridine and aniline, ${ }^{10,11}$ and for the ${ }^{1} \mathrm{H}-{ }^{13} \mathrm{C}$ coupling in $o$-dichlorobenzene. ${ }^{12}$ In 1995 , the field-induced effect on the ortho ${ }^{1} \mathrm{H}-{ }^{1} \mathrm{H}$ coupling in benzene and halobenzenes was reported by Laatikainen et al. ${ }^{13}$ and, for the ${ }^{15} \mathrm{~N}$ chemical shift in a protein-DNS complex, by Ottiger, Tjandra and Bax. ${ }^{14}$

For this study, we recorded the ${ }^{2} \mathrm{H},{ }^{2} \mathrm{H}-\left\{{ }^{1} \mathrm{H}\right\},{ }^{13} \mathrm{C}$ and ${ }^{13} \mathrm{C}-\left\{{ }^{1} \mathrm{H}\right\}$ NMR spectra of $1,3,5-$ $\mathrm{D}_{3}$-benzene at four different magnetic fields. From these spectra we reveal information about (a) the orientational order arising from the interaction of the magnetic field with the anisotropy of the diamagnetic susceptibility tensor of benzene, (b) the ${ }^{2} \mathrm{H}$ quadrupole splitting and its sign, and (c) the apparent magnetic-field dependence of the one-bond ${ }^{13} \mathrm{C}-{ }^{1} \mathrm{H}$ and ${ }^{13} \mathrm{C}-{ }^{2} \mathrm{H} J$ couplings. Our findings indicate that, indeed, the $J$ couplings contain a significant contribution from the respective dipolar coupling at the present field strengths. When this part is stripped out, i.e., the coupling values at zero magnetic field are determined, the ${ }^{1} \mathrm{H} /{ }^{2} \mathrm{H}$ isotope effect on the one-bond $J$ coupling is revealed.

\section{Experimental}

The sample of ca. $0.8 \mathrm{ml} 1,3,5-\mathrm{D}_{3}$-benzene was prepared into $5 \mathrm{~mm}$ (o.d.) NMR tube and degassed by a two freeze-pump-thaw cycles. Small amount of $\mathrm{CH}_{4}$ gas was added to act as the ${ }^{1} \mathrm{H}_{-}{ }^{13} \mathrm{C}$ spinspin coupling reference. Eventually the sample tube was sealed with a flame. The NMR spectra were measured on Bruker DPX200, AVANCE III 400, AVANCE III 600 and AVANCE III 800 (equipped with a TCI cryoprobe) spectrometers, which have ${ }^{1} \mathrm{H}$ resonance frequencies of 200, 400, 600 and 800 $\mathrm{MHz}$, respectively. In addition to ${ }^{1} \mathrm{H}$ spectra, ${ }^{13} \mathrm{C}$ and ${ }^{2} \mathrm{H}$ spectra were measured both with and without broadband proton decoupling. All the experiments were carried out at $300 \mathrm{~K}$. The temperature was calibrated using the standard calibration sample of ethylene glycol. The ${ }^{13} \mathrm{C}$ spectra were analyzed with PERCH, ${ }^{15}$ while the ${ }^{2} \mathrm{H}$ quadrupole splittings were determined from the ${ }^{13} \mathrm{C}$ satellites in the ${ }^{2} \mathrm{H}$ $\left\{{ }^{1} \mathrm{H}\right\}$ spectra using line shape analysis on the $\mathrm{dmfit}^{16}$ program. 


\section{Results and discussion}

The proton-coupled ${ }^{13} \mathrm{C}$ NMR spectrum of 1,3,5-D 3 -benzene consists of a doublet of triplets and a triplet of multiplets due to the ${ }^{13} \mathrm{C}-{ }^{1} \mathrm{H}$ and ${ }^{13} \mathrm{C}-{ }^{2} \mathrm{H}$ interactions, as shown in Figure 1 . Figure 1 also shows the proton-decoupled ${ }^{2} \mathrm{H}$ spectrum revealing the doublet splitting due to the ${ }^{2} \mathrm{H}$ quadrupole coupling. On the contrary, the ${ }^{13} \mathrm{C}-\left\{{ }^{1} \mathrm{H}\right\}$ NMR spectrum consists of a singlet and a triplet of quintets. The doublet splitting is due to the one-bond ${ }^{13} \mathrm{C}-{ }^{1} \mathrm{H}$ interaction, $\Delta_{\mathrm{CH}}$, which is shown, as an example, in Figure 2 as a function of the magnetic flux density at $300 \mathrm{~K}$. Generally, the doublet and triplet splittings are considered to be due to the respective one-bond scalar $J$ couplings. Here we use the $\Delta$ symbols for the reason explained below. The dependence of $\Delta_{\mathrm{CH}}$ on $B$ may result (a) from the direct field dependence of the electronic property of $J_{\mathrm{CH}}$ coupling or (b) indirectly via the dependence of the orientational order parameter $S$ (vide infra) on the magnetic field. The latter renders visible the anisotropic contributions, dipole-dipole coupling $\left(D_{\mathrm{CH}}\right)$ and/or the spin-spin coupling, $J_{\mathrm{CH}}^{\text {aniso }}$, which average out in non-oriented liquids. The direct field dependence of $J_{\mathrm{CH}}$ is hardly of observable magnitude and it was ruled out in the present study by using ${ }^{13} \mathrm{CH}_{4}$ as an internal reference. The $J_{\mathrm{CH}}$ parameter of ${ }^{13} \mathrm{CH}_{4}$ was measured at three fields and was found to be constant, $J_{\mathrm{CH}}=125.668 \pm 0.001$ Hz. As methane is a molecule that, on account of its symmetry, is not oriented by magnetic field (its diamagnetic susceptibility is zero), the constancy of $J_{\mathrm{CH}}$ as a function of $B$ implies that any apparent field dependence of the $\Delta_{\mathrm{CH}}$ parameter arises solely via the indirect mechanism also in $1,3,5-\mathrm{D}_{3}$ benzene. 


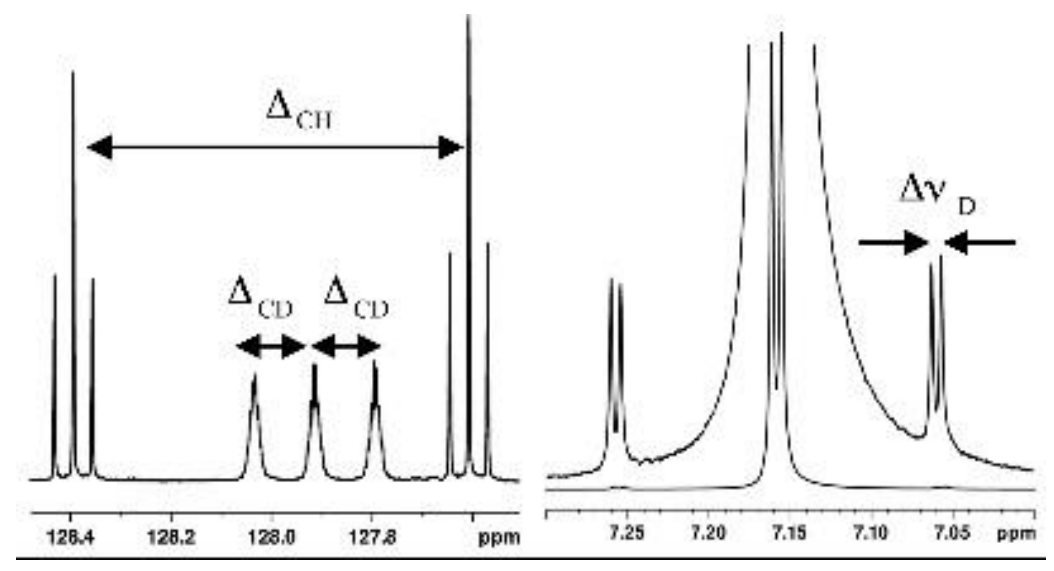

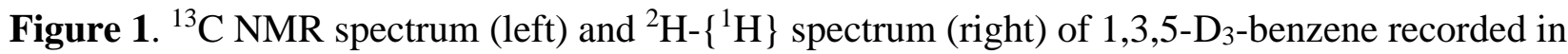
the magnetic field of $18.7829 \mathrm{~T}$ (proton Larmor frequency $800 \mathrm{MHz}$ ). The extracted quantities are defined in the spectra.

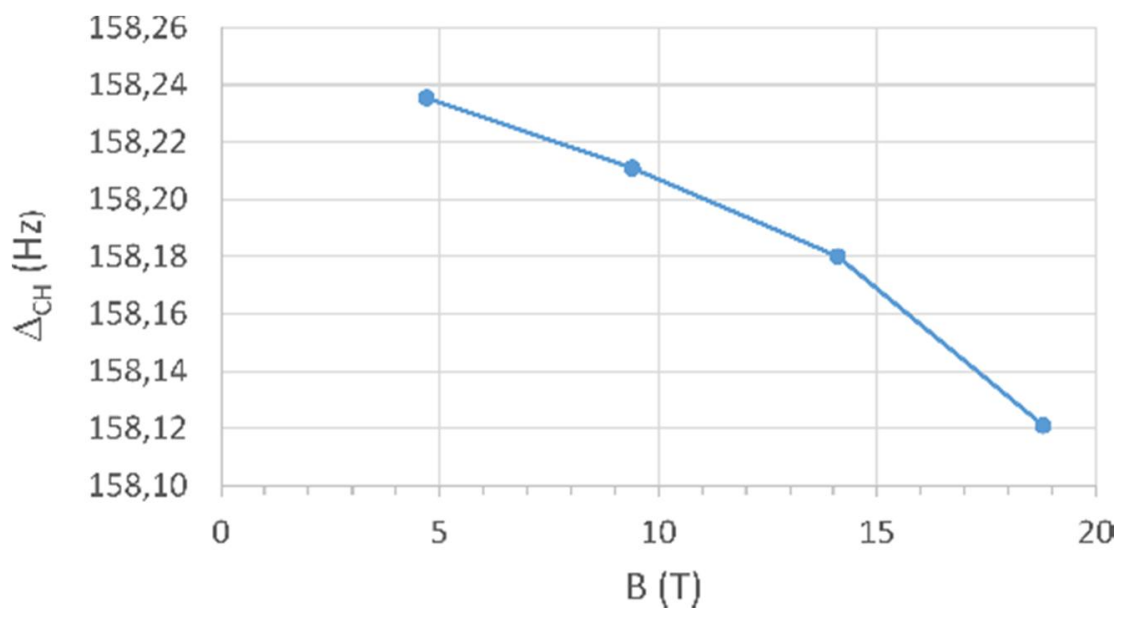

Figure 2. The splitting $\Delta_{\mathrm{CH}}$ in 1,3,5- $\mathrm{D}_{3}$-benzene as a function of the magnetic flux density.

In 1,3,5-D 3 -benzene, similar field dependence is seen for the one-bond ${ }^{2} \mathrm{H}_{-}{ }^{13} \mathrm{C}$ splitting and ${ }^{2} \mathrm{H}$ quadrupole splitting as for $\Delta_{\mathrm{CH}}$. Obviously, the reason for the observed field dependence is the slight orientation of the $1,3,5-\mathrm{D}_{3}$-benzene molecule, in the formally isotropic medium. This phenomenon was recently reported for $\mathrm{D}_{6}$-benzene and $\mathrm{D}$-chloroform at very high magnetic fields. ${ }^{17,18}$ Consequently, a plausible explanation for the observed behavior shown in Figure 2 is a small contribution from the direct ${ }^{13} \mathrm{C}-{ }^{1} \mathrm{H}$ coupling, $D_{\mathrm{CH}}$, and (possibly) from $J_{\mathrm{CH}}^{\text {aniso }}$ due to the anisotropy of the spin-spin coupling tensor. 
The dipolar couplings between a pair of nuclei in the benzene ring can be presented in the form

$D_{K L}=-\frac{\mu_{o} \hbar \gamma_{\mathrm{K}} \gamma_{\mathrm{L}}}{8 \pi^{2}} \frac{1}{r_{K L}^{3}} S_{\perp}=K_{K L} \frac{1}{r_{K L}^{3}} S_{\perp}$

Here, $S_{\perp}$ is the orientational order parameter perpendicular to the symmetry axis of benzene, while the other symbols have their usual meanings. One should note that in some earlier papers, the definition of $D_{K L}$ differs by a factor of 2 from that in Eq. (1), i.e., the present values should be multiplied by 2 when compared with the earlier ones.

The contribution from the anisotropy of the spin-spin coupling tensor $\boldsymbol{J}_{\mathrm{KL}}$ is

$J_{K L}^{\text {aniso }}=\frac{2}{3} \Delta J_{K L} S_{\|}$,

where $\Delta J_{K L}=J_{K L, \|}-J_{K L, \perp}$ is the anisotropy relative to the symmetry axis of the axially symmetric molecule and $S_{\|}$is the orientational order parameter of the symmetry axis. These two contributions cannot be measured separately but as a combination

$D_{K L}^{\exp }=D_{K L}+\frac{1}{2} J_{K L}^{\text {aniso }}$

The anisotropy of the one-bond ${ }^{1} \mathrm{H}-{ }^{13} \mathrm{C}$ spin-spin coupling tensor is small. There are no experimental results available but computations indicate that $\Delta^{1} \boldsymbol{J}_{\mathrm{CH}}$ is only a few $\mathrm{Hz}$ at maximum. ${ }^{19,20,21}$ Moreover, $S_{\|}$is small, as shown below and, therefore, the term $\frac{1}{2} J_{\mathrm{CH}}^{\mathrm{aniso}}$ is negligible and can be omitted. One should note that $J_{K L}^{\text {aniso }}$ may not always be negligible in the case of heavier nuclei. ${ }^{21,22}$

The orientational order parameters, $S_{\|}$and $S_{\perp}$, which arise from the interaction between the magnetic field and the anisotropy of the diamagnetic susceptibility of benzene, can be obtained using 
Boltzmann statistics. The component of the diamagnetic susceptibility in the direction of the magnetic field, which defines the $Z$ axis of the laboratory frame, is

$\chi_{Z Z}=\chi^{\text {iso }}+\frac{2}{3} \Delta \chi P_{2}(\cos \theta)$,

where $\chi^{\text {iso }}$ is the isotropic average and $\Delta \chi=\chi_{\|}-\chi_{\perp}$ is the anisotropy of the cylindrically symmetric susceptibility tensor in the molecule-fixed frame, and $P_{2}(\cos \theta)$ is the second Legendre polynomial with $\theta$ the angle between the molecular symmetry axis and the external magnetic field. The magnetic energy is, consequently,

$E_{B}=-\frac{1}{2} \boldsymbol{B} \cdot \chi \cdot \boldsymbol{B}=-\frac{1}{2} \chi_{Z Z} B^{2}$

The orientational order parameter of the symmetry axis, $S_{\|}$, is obtained as follows (one should note that the isotropic part in $\chi_{Z Z}$ can be neglected because it does not affect the orientation):

$S_{\|}=\left\langle\frac{1}{2}\left(3 \cos ^{2} \theta-1\right)\right\rangle_{B}=\frac{\int_{0}^{\pi} \frac{1}{2}\left(3 \cos ^{2} \theta-1\right) \exp \left(\frac{\frac{1}{3} \Delta \chi P_{2}(\cos \theta) B^{2}}{k T}\right) \sin \theta d \theta}{\int_{0}^{\pi} \exp \left(\frac{\frac{1}{3} \Delta \chi P_{2}(\cos \theta) B^{2}}{k T}\right) \sin \theta d \theta} \approx \frac{\Delta \chi}{15 k T} B^{2}$.

In Eq. (6), $k$ is the Boltzmann constant and $T$ the temperature. The exponent $\frac{\frac{1}{3} \Delta \chi P_{2}(\cos \theta) B^{2}}{k T}$ is of the order of $10^{-5}-10^{-4}$ at the magnetic fields and temperature used in the present study and, consequently, the exponential function can be replaced by a series expansion. The simple final form in Eq. (6) is obtained when higher than the second-order terms in $B$ are omitted. The order parameter in the plane of benzene thus becomes 
$S_{\perp}=P_{2}\left(\cos 90^{\circ}\right) S_{\|}=-\frac{1}{2} \frac{\Delta \chi}{15 k T} B^{2}$

The experimentally detected splittings due to the ${ }^{1} \mathrm{H}-{ }^{13} \mathrm{C}$ and ${ }^{2} \mathrm{H}-{ }^{13} \mathrm{C}$ interactions are sums of the respective $J$ and $D$ couplings, i.e., $\Delta=J+2 D$. Thus, substitution of the right-hand side of Eq. (7) for $S_{\perp}$ in Eq. (1) results in the following equation:

$\Delta_{\mathrm{CX}}=J_{\mathrm{CX}}(0)+2 D_{\mathrm{CX}}=J_{\mathrm{CX}}(0)+\frac{\mu_{o} \hbar \gamma_{\mathrm{C}} \gamma_{\mathrm{X}}}{8 \pi^{2}} \frac{1}{r_{\mathrm{CH}}^{3}} \frac{\Delta \chi}{15 k T} B^{2}$,

where $\mathrm{X}=\mathrm{H}$ or $\mathrm{D}$, indicating linear dependence of the observed splittings on $B^{2}$. The $\Delta_{\mathrm{CH}}$ and $\Delta_{\mathrm{CD}}$ parameters are dominated by the respective one-bond $J$ couplings, which are generally known to be positive. Thus the sign of $\Delta_{\mathrm{CX}}$ is positive, too. In the least-squares fit, the C-H bond length was fixed to $r_{\mathrm{CH}}=1.0841 \AA$, while the C-D bond length was fixed to $1.0830 \AA{ }^{23}$ The third field-dependent quantity, the ${ }^{2} \mathrm{H}$ quadrupole splitting, is

$\Delta v_{\mathrm{D}}=\frac{3}{2} q_{z z}(1+\eta) S_{\perp}=\frac{3}{2} q_{z z}(1+\eta)\left(-\frac{1}{2} \frac{\Delta \chi}{15 k T} B^{2}\right)=-\frac{3}{4} \frac{q_{z z}(1+\eta) \Delta \chi}{15 k T} B^{2}$,

where $q_{z z}$ is the ${ }^{2} \mathrm{H}$ quadrupole coupling tensor component in the C-D bond direction and $\eta$ is the asymmetry parameter of the tensor, defined as

$\eta=\frac{q_{x x}-q_{y y}}{q_{z z}}$

Eqs. (8) and (9) contain a common parameter, the susceptibility anisotropy $\Delta \chi$. Consequently, the joint least-squares fit of functions (8)-(9) to the experimental splittings is based on the adjustable parameters $\Delta \chi, J_{\mathrm{CH}}(0), J_{\mathrm{CD}}(0), q_{z z}$, and $\eta$. The results are shown in Table 1 and Figs. 3 and 4.

Table 1. Results from the joint fit of Eqs. (8)-(9) to the experimental splittings $\Delta_{\mathrm{CH}}, \Delta_{\mathrm{CD}}$ and $\Delta v_{\mathrm{D}}$. 


\begin{tabular}{|c|c|c|}
\hline Parameter & Fitted value & Literature value / ref. \\
\hline$\Delta \chi$ & $(-889.5 \pm 0.2) \times 10^{-30} \mathrm{JT}^{-2}$ & $-1026 \times 10^{-30} \mathrm{JT}^{-2} / 11$ \\
\hline$J_{\mathrm{CH}}(0)$ & $158.244 \pm 0.003 \mathrm{~Hz}$ & $158.380 \mathrm{~Hz} / 13$ \\
\hline$J_{\mathrm{CD}}(0)$ & $24.247 \pm 0.001 \mathrm{~Hz}$ & $24.267 \pm 0.006 \mathrm{~Hz} / 24$ \\
& & \\
\hline$q_{z z}$ & $185.940 \pm 0.003 \mathrm{kHz}$ & $184.7 \pm 0.3 \mathrm{kHz} / 28$ \\
\hline$\eta$ & $0.054 \pm 0.001$ & $0.056 / 28$ \\
\hline
\end{tabular}
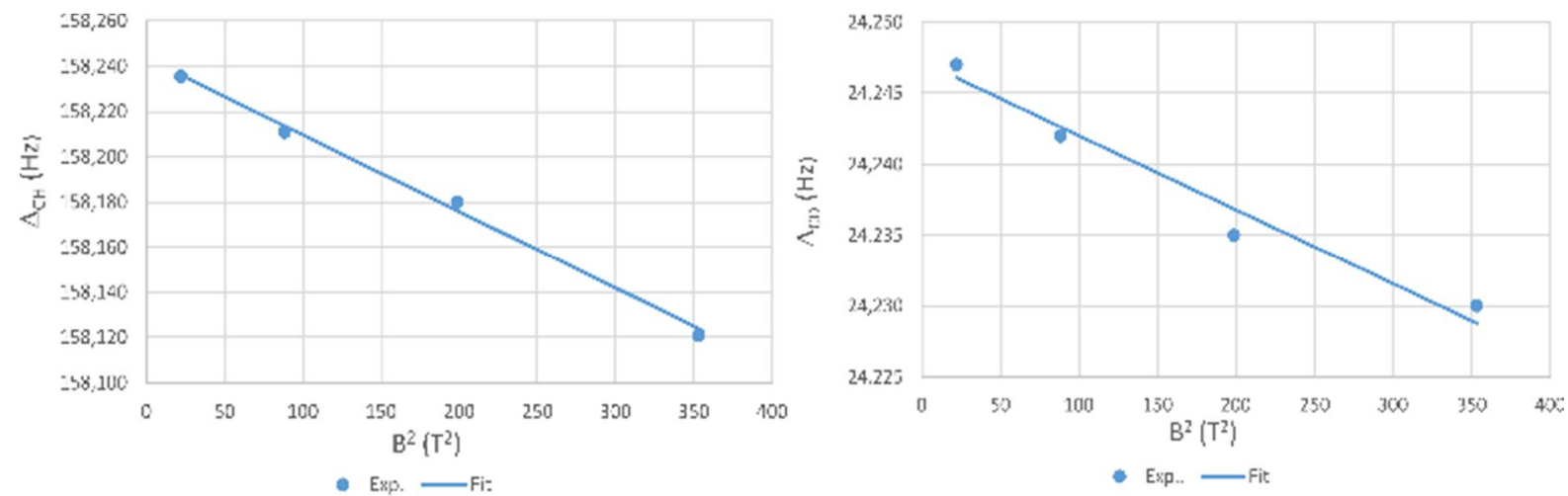

Figure 3. The splittings $\Delta_{\mathrm{CH}}$ and $\Delta_{\mathrm{CD}}$ as a function of the square of the magnetic flux density.

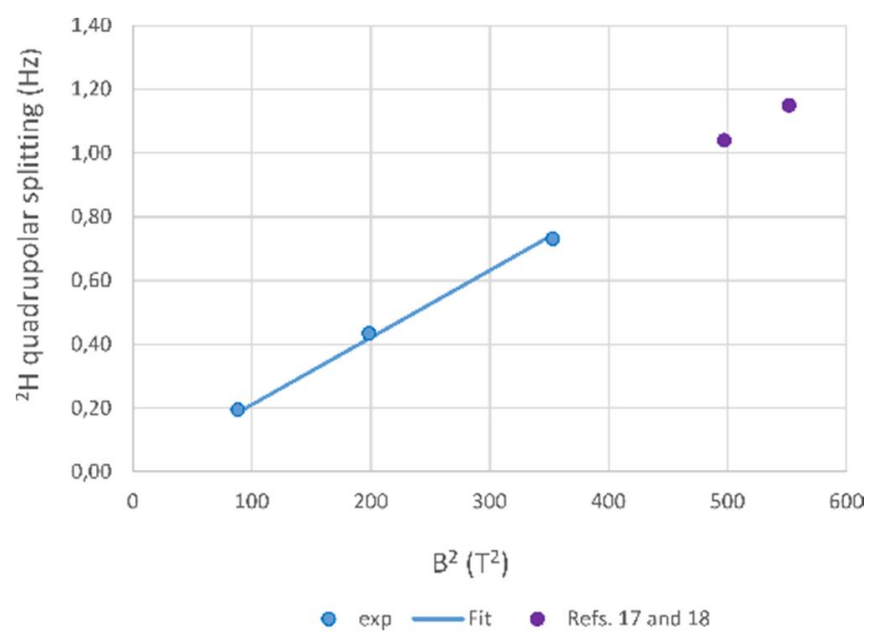


Figure 4. ${ }^{2} \mathrm{H}$ quadrupole splitting, $\Delta v_{\mathrm{D}}$, as a function of the square of the magnetic flux density. The two points at the highest fields are from refs. 17 and 18 , for $\mathrm{C}_{6} \mathrm{D}_{6}$. The least-squares fit is based on the three lower field points only.

The $\Delta \chi$ value of $-889.5 \times 10^{-30} \mathrm{JT}^{-2}$ results in the orientational order parameter of the benzene symmetry axis of

$S_{\|}=-1.432 \times 10^{-8} B^{2}\left(\frac{1}{\mathrm{~T}^{2}}\right)$

where T refers to the SI unit Tesla of the magnetic flux density.

First-principles calculations of the susceptibility tensor were performed using the Dalton 2015 program..$^{25}$ First, the susceptibility tensor at the benzene equilibrium geometry of Gauss and Stanton ${ }^{22}$ $\left(r_{\mathrm{CC}}=1.3914 \AA ; r_{\mathrm{CH}}=1.0802 \AA\right)$ was computed using the second-order polarization propagator with coupled-cluster singles and doubles amplitudes [SOPPA(CCSD)] method ${ }^{26}$ with the cc-pCV5Z basis set. $^{27}$ The results at this level for the isotropic susceptibility $\chi$ and susceptibility anisotropy $\Delta \chi$ are -883.3 and -1010.1 , respectively, in units of $10^{-30} \mathrm{~J} / \mathrm{T}^{2}$. To these equilibrium geometry values we can add rovibrational contributions at $300 \mathrm{~K}$ using the same method as employed earlier for the corresponding contributions to the deuterium quadrupole coupling tensor ${ }^{28}$ and susceptibility ${ }^{17}$ in benzene. Carrying out these corrections at the density-functional theory (DFT) level using the 3parameter hybrid exchange-correlation functional $\mathrm{B}^{2} \mathrm{LYP}^{29}$ and the cc-pCVTZ basis ${ }^{26}$ leads, at 300 $\mathrm{K}$, to the final values of -871.0 and -973.5 (in units of $10^{-30} \mathrm{~J} / \mathrm{T}^{2}$ ) for $\chi$ and $\Delta \chi$, respectively. These results are obtained as the sum of the SOPPA(CCSD) data at the equilibrium geometry and the DFTbased rovibrational corrections. The fact that a roughly $10 \%$ larger absolute value of $\Delta \chi$ is obtained from the computations than from the joint fit to the experimental data described above, is due to deficiencies in the present electronic structure treatment (electron correlation and basis set) and, as the main source of imprecision, the lack of solvent/medium corrections in the theoretical calculations, 
when comparing to liquid-state experiments. Other recent, entirely DFT-based computations led to the values of $\Delta \chi$ equal to $-1056 \times 10^{-30} \mathrm{~J} / \mathrm{T}^{2},{ }^{17}$ at the B3LYP/aug-cc-pVTZ level including rovibrational corrections at $300 \mathrm{~K}$ for the perdeuterated isotopomer) and $-1065 \times 10^{-30} \mathrm{~J} / \mathrm{T}^{2}$ (B3LYP/aug-cc-pVTZ without rovibrational corrections). ${ }^{18}$

The joint fit results in the one-bond ${ }^{1} \mathrm{H}-{ }^{13} \mathrm{C}$ spin-spin coupling constant at zero magnetic field of $J_{\mathrm{CH}}(0)=158.244 \pm 0.003 \mathrm{~Hz}$. Jackowski, Maciaga and Wilczek measured the value of 157.78 $\pm 0.02 \mathrm{~Hz}$ at $11.7 \mathrm{~T}$ for gaseous benzene. ${ }^{30}$ (We note in passing that this value may include a contribution from $2 D_{\mathrm{CH}}$, of about $-0.10 \mathrm{~Hz}$, as estimated on the basis of the present results.) It can be observed from comparison to our present liquid-state data that the coupling increases by about 0.4 $\mathrm{Hz}$ at the gas-to-liquid phase transition. The corresponding $J_{\mathrm{CD}}(0)$ is $24.247 \pm 0.001 \mathrm{~Hz}$. The ratio $J_{\mathrm{CH}}(0) / J_{\mathrm{CD}}(0)$ thus equals $6.5262 \pm 0.008 \%$ differing from the gamma ratio $\gamma_{\mathrm{H}} / \gamma_{\mathrm{D}}=6.51439804$ (Ref. 31) significantly, by $0.18 \%$. The deviation of the ratio of the couplings from the gamma ratio suggests the existence of an isotope effect. The primary isotope effect of the one-bond ${ }^{1} \mathrm{H}-{ }^{13} \mathrm{C}$ coupling is generally defined as ${ }^{32}$

$\Delta_{\mathrm{p}}{ }^{1} J\left({ }^{13} \mathrm{C}^{2 / 1} \mathrm{H}\right) \equiv{ }^{1} J_{\mathrm{CD}} \frac{\gamma_{\mathrm{H}}}{\gamma_{\mathrm{D}}}-{ }^{1} J_{\mathrm{CH}}$.

In the present case the value $-0.288 \pm 0.009 \mathrm{~Hz}$ is obtained for $\Delta_{\mathrm{p}}{ }^{1} J\left({ }^{13} \mathrm{C}^{2 / 1} \mathrm{H}\right)$ at zero magnetic field. The negative sign seems to be general for this kind of effects. ${ }^{32}$

In fact, the primary isotope effect is, in principle, dependent on the magnetic field. Eq. (12) can be presented in the more complete form

$\Delta_{\mathrm{p}}{ }^{1} J\left({ }^{13} \mathrm{C}^{2 / 1} \mathrm{H}\right)=\left({ }^{1} J_{\mathrm{CD}}+2 D_{\mathrm{CD}}\right) \frac{\gamma_{\mathrm{H}}}{\gamma_{\mathrm{D}}}-\left({ }^{1} J_{\mathrm{CH}}+2 D_{\mathrm{CH}}\right)$, 
including the corresponding direct coupling contributions. Using the definition of Eq. (1) for the $D$ coupling, Eq. (13) becomes

$\Delta_{\mathrm{p}}{ }^{1} J\left({ }^{13} \mathrm{C}^{2 / 1} \mathrm{H}\right) \equiv{ }^{1} J_{\mathrm{CD}} \frac{\gamma_{\mathrm{H}}}{\gamma_{\mathrm{D}}}-{ }^{1} J_{\mathrm{CH}}+2 D_{\mathrm{CH}}\left[\left(\frac{r_{\mathrm{CH}}}{r_{\mathrm{CD}}}\right)^{3}-1\right]$.

In the present case, $2 D_{\mathrm{CH}}$, which is proportional to $B^{2}$, obtains values between -7.5 and $-120 \mathrm{mHz}$ depending on the strength of the magnetic field, and the third power of the ratio of the bond lengths is 1.00305. Consequently, the effect of the direct dipolar coupling term in Eq. (14) is negligible in the present case.

As was concluded above, the order parameter $S_{\|}$is negative and, due to the tracelessness of the orientation tensor, its component $S_{\perp}$ is, correspondingly, positive. If the signs are not known, they can be determined from the proton-coupled ${ }^{2} \mathrm{H}$ NMR spectra, as shown below. Namely, the dipolar couplings can be calculated from Eq. (1) when the internuclear distances and orientational order parameter are known. From Eqs. (1) and (9) we can further conclude that the $D_{K L}$ couplings and ${ }^{2} \mathrm{H}$ quadrupole splitting $\Delta v_{\mathrm{D}}$ must possess opposite signs. This conclusion follows from the opposite signs of $q_{\mathrm{CD}}(>0)$ and $K_{K L}(<0)$, while both $D_{K L}$ and $\Delta \nu_{\mathrm{D}}$ are dependent on the same orientational order parameter, $S_{\perp}$. The estimate for the magnitude of the latter order parameter is obtained from Eq. (9) using $\Delta v_{\mathrm{D}}=0.73 \mathrm{~Hz}$ from the ${ }^{2} \mathrm{H}$ NMR spectrum at $800 \mathrm{MHz}, q_{z z}=184.7 \mathrm{kHz}$ and $\eta=0.056 .{ }^{27}$ The ${ }^{2} \mathrm{H}$ spectra were simulated on the PERCH program ${ }^{15}$ using two sign combinations: (1) $D_{K L}(K, L$ $={ }^{1} \mathrm{H},{ }^{2} \mathrm{H}$ ) couplings are negative and $\Delta v_{\mathrm{D}}$ positive, and (2) $D_{K L}$ couplings are positive and $\Delta v_{\mathrm{D}}$ negative. The resulting spectra are compared with the experimental spectrum (taken at $800 \mathrm{MHz}$ ) in Fig. 5. The internuclear distances and the $K_{K L}$ factors are shown in Table 2 . The $J$ couplings, in turn, are from $\mathrm{C}_{6} \mathrm{H}_{6}$ (see ref. 13) scaled by the gyromagnetic ratios. Table 3, in turn, lists the dipolar couplings. 
Table 2. D-D and D-H internuclear distances and the $K_{K L}$ factors used in the calculation of the dipolar couplings for the simulation of the proton-coupled ${ }^{2} \mathrm{H}$ NMR spectrum shown in Fig. 5. The corresponding $J$ couplings are listed as well. ${ }^{\text {a) }}$

$$
\begin{array}{lll}
R_{\mathrm{DH}}^{o}=2.47 \AA & K_{\mathrm{HH}}=-120120 \mathrm{~Hz}^{3} & J_{\mathrm{HD}}^{o}=1.158 \mathrm{~Hz} \\
R_{\mathrm{DD}}^{m}=4.28 \AA & K_{\mathrm{DH}}=-18440 \mathrm{~Hz} \AA^{3} & J_{\mathrm{DD}}^{m}=0.032 \mathrm{~Hz} \\
R_{\mathrm{DH}}^{p}=4.94 \AA & K_{\mathrm{DD}}=-2830.6 \mathrm{~Hz}^{3} & J_{\mathrm{HD}}^{p}=0.100 \mathrm{~Hz} \\
R_{\mathrm{HH}}^{m}=4.28 \AA & & J_{\mathrm{HH}}^{m}=1.373 \mathrm{~Hz}
\end{array}
$$

a) The symbols $o, p$ and $m$ refer to ortho, meta and para, respectively.

Table 3. The magnitudes of the dipolar couplings in 1,3,5- $\mathrm{D}_{3}$-benzene at variable magnetic fields $B$. The $D$ couplings are in $\mathrm{mHz}$ while $B$ values are in Tesla. ${ }^{\text {a) }}$

\begin{tabular}{rrrrr}
\hline \multicolumn{1}{l}{$B$} & $o-D_{\mathrm{DH}}$ & $m-D_{\mathrm{DD}}$ & $p-D_{\mathrm{DH}}$ & $m-D_{\mathrm{HH}}$ \\
\hline 18.79 & 3.09 & 0.09 & 0.39 & 3.88 \\
14.09 & 1.74 & 0.05 & 0.22 & 2.18 \\
9.40 & 0.77 & 0.02 & 0.10 & 0.96 \\
4.70 & 0.19 & 0.00 & 0.02 & 0.24 \\
\hline
\end{tabular}

a) For the meaning of $o, p$, and $m$, see footnote in Table 2 . 

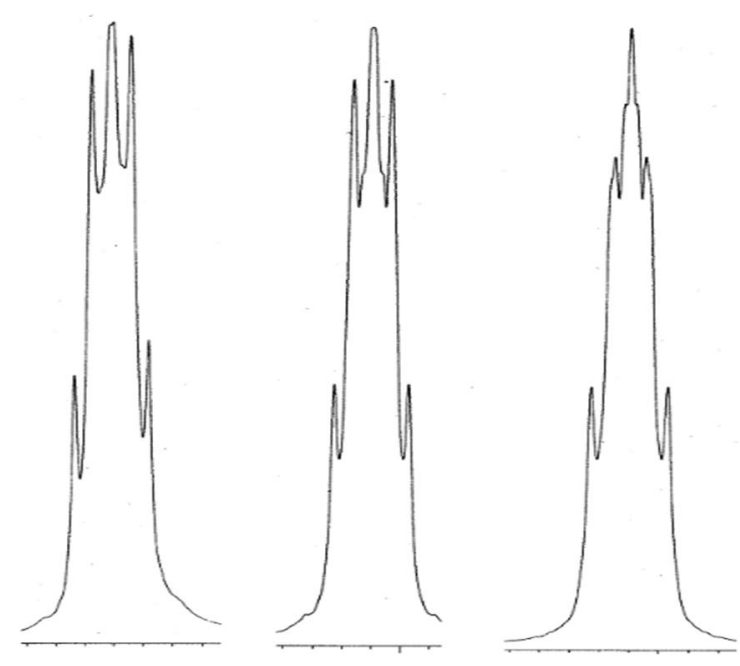

Figure 5. Experimental and simulated ${ }^{2} \mathrm{H}$ NMR spectra of 1,3,5- $\mathrm{D}_{3}$-benzene oriented by magnetic field. Left: Experimental spectrum measured on an $800 \mathrm{MHz}$ spectrometer. Mid: simulated spectrum with positive ${ }^{2} \mathrm{H}$ quadrupole splitting and negative dipolar couplings. Right: simulated spectrum with negative ${ }^{2} \mathrm{H}$ quadrupole splitting and positive dipolar couplings.

Figure 5 clearly proves that the ${ }^{2} \mathrm{H}$ quadrupole splitting must be positive and the dipolar couplings negative. This further means that the orientational order parameter in the benzene plane, i.e., in the perpendicular direction relative to the symmetry axis, must be positive. It is important to note that although the dipolar couplings are small (see Table 3), they affect the fine structure of the spectrum. A nice example is given in ref. 13 in which a very high-resolution spectrum of benzene is analyzed.

\section{Conclusions}

Isotropic liquids are defined as being uniform in all directions. In such environments the NMR tensors average to their isotopic values, equal to one third of the trace of the tensor. This means that the anisotropic parts of the corresponding tensors vanish. However, this situation breaks down in cases where the anisotropy of the diamagnetic susceptibility of the molecules is large and the NMR experiments are performed at high magnetic fields. The interaction of the magnetic flux density with 
the diamagnetic susceptibility leads to a slightly anisotropic orientational distribution of the molecules and, consequently, the NMR tensors include non-zero anisotropic contributions. Presently, efforts are made toward higher and higher magnetic fields, meaning that both the directly fieldinduced and indirectly field-dependent anisotropic contributions are becoming more pronounced. In this study, we have shown that already at the magnetic field of $4.7 \mathrm{~T}$, the dipolar contribution to the

one-bond ${ }^{1} \mathrm{H}_{-}{ }^{13} \mathrm{C}$ coupling in benzene exceeds the generally reported error limits. The application of high magnetic field in liquid-state NMR experiments is generally considered beneficial. As shown here, the emerging anisotropic contributions affect the accuracy of the extracted NMR parameters.

\section{Acknowledgements}

Dr. R. Seydoux, Bruker Biospin, Fällanden, Switzerland, is thanked for the data obtained on an 800 MHz spectrometer. CSC-IT Center for Science, Ltd., and the Finnish Grid and Cloud Infrastructure provided the computational resources. 


\section{References}

[1] N.F. Ramsey, Possibility of Field Dependent Nuclear Magnetic Shielding, Phys. Rev, E 1 (1970) 1320.

[2] M.R. Bendall and D.M. Doddrell, Measurement of the Field Dependent Chemical Shifts, J. Magn. Reson., 3 (1979) 659.

[3] P. Manninen and J. Vaara, Magnetic-field dependence of ${ }^{59} \mathrm{Co}$ nuclear magnetic shielding in Co(III) compounds Phys. Rev. A, 69 (2004) 022503.

[4] J. Boyd, G.I. Pagola, C. Caputo, M.B. Ferraro and P. Lazzeretti, Calculation of Hypershielding Contribution to Isotropic Nitrogen Shielding in Strong Magnetic Fields, J. Chem. Theory Comput. 5 (2009) 1343.

[5] W.T. Raynes and S.J. Stevens, Magnetic-field dependent nuclear spin-spin coupling, Magn. Reson. Chem. 30 (1992) 124.

[6] T. Meersmann and M. Haake, Magnetic Field Dependent Xenon-131 Quadrupolar Splitting in Gas and Liquid Phase NMR, Phys. Rev. Lett. 81 (1998) 1211.

[7] J. Vaara and P. Pyykkö, Magnetic-Field-Induced Quadrupole Splitting in Gaseous and Liquid ${ }^{131}$ Xe NMR: Quadratic and Quartic Field Dependence, Phys. Rev. Lett., 86 (2001) 3268.

[8] P. Manninen, J. Vaara and P. Pyykkö, Magnetic-field-induced quadrupole coupling in the nuclear magnetic resonance of noble-gas atoms and molecules, Phys. Rev. A, 70 (2004) 043401.

[9] N. Abuzaid, A.M. Kantola and J. Vaara, Magnetic field-induced quadrupole coupling in atomic ${ }^{131}$ Xe, Molec. Phys., 111 (2013) 1390.

[10] P.C.M. van Zijl, B.H. Ruessink, J. Bulthuis and C. MacLean, NMR of Partially Aligned Liquids: Magnetic Susceptibility Anisotropies and Dielectric Properties, Acc. Chem. Res., 17 (1984) 172.

[11] P.C.M. van Zijl, C. MacLean and A.A. Bothner-By, Angular correlation and diamagnetic susceptibilities studied by high field NMR, J. Chem. Phys., 83 (1985) 4410.

[12] F. Anet, Magnetic Alignment Effects in the 500-MHz 'H NMR Spectrum of oDichlorobenzene in Acetone-d6, J. Am. Chem. Soc., 108 (1986) 1354.

[13] R. Laatikainen, J. Ratilainen, R. Sebastian and H. Santa, NMR study of aromatic-aromatic interactions for benzene and some other fundamental aromatic systems using alignment of aromatics in strong magnetic fields, J. Am. Chem. Soc., 117 (1995) 11006.

[14] M. Ottiger, N. Tjandra and A. Bax, Magnetic Field dependent Amide ${ }^{15} \mathrm{~N}$ Chemical Shifts in a Protein-DNA Complex Resulting from Magnetic Ordering in Solution, J. Am. Chem. Soc., 119 (1997) 9825.

[15] R. Laatikainen, M. Niemitz, J. Sundelin and T. Hassinen, PERCH project, Department of Chemistry, University of Kuopio, Finland. PERCH solutions is presently owned by Bruker BioSpin.

[16] D. Massiot, F. Fayon, M. Capron, I. King, S. Le Calvé, B. Alonso, J.O. Durand, B. Bujoli, Z. Gan and G. Hoatson, Modelling one- and two-dimensional solid-state NMR spectra, Magn. Reson. Chem., 40 (2002) 70.

[17] L.M. Heist, C.-D. Poon, E.T. Samulski, D.J. Photinos, J. Jokisaari, J. Vaara, J.W. Emsley, S. Mamone and M. Lelli, Benzene at $1 \mathrm{GHz}$. Magnetic field-induced fine structure, J. Magn. Reson., 258 (2015) 17.

[18] E.T. Samulski, C.-H. Poon, L.M. Heist and D.J. Photinos, Liquid-state structure via very high-field nuclear magnetic resonance discriminates among force fields, J. Phys. Chem. Lett., 6 (2015) 3626.

[19] J. Kaski, J. Vaara and J. Jokisaari, ${ }^{13} \mathrm{C}-{ }^{13} \mathrm{C}$ spin-spin coupling tensors in benzene as determined experimentally by liquid crystal NMR and theoretically by ab initio calculations, 
J. Am. Chem. Soc., 118 (1996) 8879.

[20] J. Lounila and J. Jokisaari, Anisotropies in spin-spin coupling constants and chemical shifts as determined from the NMR spectra of molecules oriented by liquid crystals solvents, Progr. NMR Spectroscopy, 15 (1982) 249.

[21] J. Vaara, J. Jokisaari, R.E. Wasylishen and D.L. Bryce, Spin-spin coupling tensors as determined by experiment and computational chemistry, Progr. NMR Spectroscopy, 41 (2002) 233.

[22] J. Jokisaari and J. Vaara, Nuclear spin-spin coupling anisotropy in van der Waals-bonded ${ }^{129} \mathrm{Xe}$ dimer, Phys. Chem. Chem. Phys. 15 (2013) 11427.

[23] These are $r_{\alpha}$ bond lengths at $300 \mathrm{~K}$. They were obtained using the equilibrium $\mathrm{r}_{\mathrm{CH}}$ reported in J. Gauss and J.F. Stanton, The Equilibrium Structure of Benzene, J. Phys. Chem. A, 104 (2000) 2865 with adding the stretches due to anharmonic vibrations calculated on the B3LYP/ccpCVTZ level. They are 0.0039 and $0.0028 \AA$ for the C-H and C-D distances, respectively.

[24] A. Roznyatovsky, N.M. Sergeyev and V.A. Chertkov, ${ }^{13} \mathrm{C},{ }^{13} \mathrm{C}$ coupling constants of isotopically perturbed molecular systems: Cyclohexane, benzene, Magn. Res. Chem., 29 (1991) 304.

[25] K. Aidas, C. Angeli, K. L. Bak, V. Bakken, R. Bast, L. Boman, O. Christiansen, R. Cimiraglia, S. Coriani, J. Cukras, P. Dahle, E. K. Dalskov, U. Ekström, T. Enevoldsen, J. J. Eriksen, P. Ettenhuber, B. Fernandez, L. Ferrighi, H. Fliegl, L. Frediani, K. Hald, A. Halkier, C. Hättig, H. Heiberg, T. Helgaker, A. C. Hennum, H. Hettema, E. Hjertenaes, S. Hoest, I.-M. Hoeyvik, M. F. Iozzi, B. Jansik, H. J. Aa. Jensen, D. Jonsson, P. Jørgensen, M. Kaminski, J. Kauczor, S. Kirpekar, T. Kjaergaard, W. Klopper, S. Knecht, R. Kobayashi, H. Koch, J. Kongsted, A. Krapp, K. Kristensen, A. Ligabue, O. B. Lutnaes, J. I. Melo, K. V. Mikkelsen, R. H. Myhre, C. Neiss, C. B. Nielsen, P. Norman, J. Olsen, J. M. H. Olsen, A. Osted, M. J. Packer, F. Pawlowski, T. B. Pedersen, P. F. Provasi, S. Reine, Z. Rinkevicius, T. A. Ruden, K. Ruud, V. Rybkin, P. Sałek, C. C. M. Samson, A. Sanchez de Meras, T. Saue, S. P. A. Sauer, B. Schimmelpfennig, K. Sneskov, A. H. Steindal, K. O. Sylvester-Hvid, P. R. Taylor, A. M. Teale, E. I. Tellgren, D. P. Tew, A. J. Thorvaldsen, L. Thoegersen, O. Vahtras, M. A. Watson, D. J. D. Wilson, M. Ziolkowski and H. Ågren, "The Dalton quantum chemistry program system", WIREs Comput. Mol. Sci., 4 (2014) 269.

[26] S.P.A. Sauer, Second-order polarization propagator approximation with coupled-cluster singles and doubles amplitudes - SOPPA(CCSD): the polarizability and hyperpolarizability of Li-, J. Phys. B., 30 (1997) 3773; Theoretical estimates of the rotational g-factor. magnetizability and electric dipole moment of GaH, Chem. Phys. Lett., 260 (1996) 271.

[27] D. E. Woon and Th. H. Dunning, Jr., Gaussian basis sets for use in correlated molecular calculations. V. Core-valence sets for boron through neon, J. Chem. Phys., 103 (1995) 4572.

[28] A. M. Kantola, S. Ahola, J. Vaara, J. Saunavaara, and J. Jokisaari, Experimental and quantumchemical determination of the ${ }^{2} \mathrm{H}$ quadrupole coupling tensor in deuterated benzenes, Phys. Chem. Chem. Phys., 9 (2007) 481.

[29] C. Lee, W. Yang, and R. G. Parr, Development of the Colle-Salvetti correlation-energy formula into a functional of the electron density, Phys. Rev. B, 37 (1988) 785; A. D. Becke, Density-functional thermochemistry. III. The role of exact exchange, J. Chem. Phys., 98 (1993) 5648; P. J. Stephens, F. J. Devlin, C. S. Ashvar, C. F. Chabalowski, and M. J. Frisch, $\mathrm{Ab}$ Initio Calculation of Vibrational Absorption and Circular Dichroism Spectra Using Density Functional Force Fields, J. Phys. Chem., 98 (1994) 11623.

[30] K. Jackowski, E. Maciaga and M. Wilczek, Investigation of the ${ }^{1} \mathrm{~J}\left({ }^{13} \mathrm{C}^{1} \mathrm{H}\right)$ spin-spin coupling and ${ }^{13} \mathrm{C}$ magnetic shielding of gaseous benzene- ${ }^{13} \mathrm{C}$, J. Mol. Structure, 101 (2005) 744.

[31] B. Smaller, E. Yasaitis and H.L. Anderson, Magnetic Moment of the Deuteron, Phys. Rev. 81 (1952) 896.

[32] C.J. Jameson and H.J. Osten, Isotope effects gn spin-spin coupling, J. Am. Chem. Soc. 108 
(1986) 2497. 